\title{
Resident productivity: trends over consecutive shifts
}

\author{
Rebecca Jeanmonod • Sara Damewood • \\ Christopher Brook
}

Received: 20 March 2009 /Accepted: 21 April 2009 /Published online: 30 May 2009

(C) Springer-Verlag London Ltd 2009

\begin{abstract}
Background It has been shown that residents' ability to see more patients and patients of higher acuity improves with level of training.

Aims No published study has reviewed whether residents become less productive with consecutive shifts. Determining peak resident productivity can optimize staffing to manage patient flow and enhance resident exposure to patients, which is critical to their education. We examine the relationship between resident productivity and number of consecutive shifts worked.

Methods This is a retrospective review of emergency medicine (EM) resident productivity defined as patients evaluated per hour per shift. Data were collected utilizing patient tracker software which provides a record of physician assignment and checked against the computerized medical record. Residents were credited with a patient if
\end{abstract}

Presented at NY regional SAEM meeting, April 2008 and at national SAEM meeting, May 2008.

The views expressed in this paper are those of the author(s) and not those of the editors, editorial board or publisher.

\author{
R. Jeanmonod $(\square)$ \\ Department of Emergency Medicine, St. Luke's Hospital, \\ 801 Ostrum St., \\ Bethlehem, PA 18015, USA \\ e-mail: rebeccajeanmonod@yahoo.com \\ S. Damewood $\cdot$ C. Brook \\ Albany Medical Center, \\ 43 New Scotland Ave., \\ Albany, NY 12208, USA \\ S. Damewood \\ e-mail: damewos@mail.amc.edu \\ C. Brook \\ e-mail: brookc@mail.amc.edu
}

they initiated the workup and dictated the chart. Productivity was tallied for 188 first-year shift strings, 303 secondyear shift strings, and 224 third-year shift strings beginning 1 November 2006. Analysis of variance (ANOVA) was used to assess for productivity differences based on the shift number, with the first shift in a series being designated "1," the second consecutive shift being designated " 2 ," and so on.

Results First-year residents saw 0.82, 0.81, and 0.91 patients per hour on consecutive shifts $\left(F_{(2,175)}=2.89, p=\right.$ 0.06 ), second-year residents saw $1.12,1.08,1.17$, and 1.28 patients per hour on consecutive shifts $\left(F_{(3,292)}=4.19, p=\right.$ 0.006 ), and third-year residents saw $1.19,1.24$, and 1.33 patients per hour on consecutive shifts $\left(F_{(2,211)}=4.08, p=\right.$ 0.02).

Conclusions Instead of tiring, residents maintain or improve productivity over consecutive shifts.

Keywords Administration - Overcrowding $\cdot$ Resident education

\section{Introduction}

For emergency medicine (EM) residents, a complete educational experience relies on amassing a body of knowledge through patient encounters. In order to function as an independent physician, a resident must be exposed to a wide variety of complaints and presentations as a physician-in-training, which is reflected in the total number of patients seen. Resident productivity (defined as the number of patients evaluated per hour) becomes an important factor in maximizing exposure to a broad range of complaints within a limited amount of time in the emergency department (ED). It has been established in the 
EM literature that resident productivity improves throughout training [1-7] and that productivity decreases consistently over the course of a shift [8]. There have been many studies looking at sleep deprivation as a major contributor to fatigue, and its association with declining technical performance as well as its adverse impact on patient care and residents' personal lives [9-11]. Some data have shown declining cognitive performance after consecutive ED night shifts [12]. A review of shift work involving nonmedical careers echos this finding, with errors increasing over consecutive night shifts, although this association was not found on consecutive day shifts [13]. This review also showed that, in the seven reviewed studies, consecutive nights resulted in improving efficiency for the tasks studied [13]. Both the USA and Europe have restricted physicianin-training work hours, citing patient and personal safety as the reason [14]. Beyond the concern for error, we sought to determine whether productivity is affected by consecutive shifts in the ED. This information could have a major impact on improving patient flow patterns in the ED and in optimizing resident scheduling to provide the greatest number of patient exposures throughout residency training.

\section{Methods}

Study design and protocol

This is a retrospective chart review of EM resident productivity by first-, second-, and third-year residents at a 65,000 patients per year volume academic tertiary care center. No resident in the study had any previous postgraduate medical training. Resident productivity was defined as patients evaluated per hour per shift. All ED resident shifts during the 3-month study period beginning on 1 November 2006 were included. Shift lengths were a combination of $9 \mathrm{~h}$, which include $8 \mathrm{~h}$ of patient care and $1 \mathrm{~h}$ of completion time (second- and third-year residents), and $12 \mathrm{~h}$, including entire $12 \mathrm{~h}$ of patient care (all three classes). We included both shift lengths in this study as our second- and third-year residents rotate through both types of shifts in a single string of consecutive shifts. For example, a single resident may work a 9 -h shift, then a 12 -h shift, then another 12 -h shift, or any given combination of these shifts.

Consecutive shifts were defined as shifts beginning on consecutive calendar days. Therefore, if a resident worked at 7 a.m. on the first calendar day of the month and returned to work at 11 p.m. on the second calendar day, those shifts were counted as consecutive. If a resident's shift ended at 8 a.m., however, and the resident returned to work $23 \mathrm{~h}$ later at 7 a.m., the shift was not counted as consecutive. The residents generally worked strings of two, three, or four shifts. There were four first-year residents who swapped with other residents and violated Residency Review Committee (RRC) work rules, working six (two residents), seven, and eight consecutive 12-h shifts. There were no other incidences of work rule violations.

Data were collected utilizing patient tracker software (VitalWorks), which provides a record of physician and time of assignment. This was checked against the computerized medical record to determine the physician who dictated the chart as well as against the residents' work schedule. Data collection was performed by five independent research assistants previously trained in accessing the patient tracker software. Ten percent of data were collected by two research assistants with $95 \%$ agreement. Data were entered in a standardized spreadsheet created for this study.

Residents were credited with a patient only if they initiated the diagnostic workup and dictated the history, physical exam, medical decision making, and plan. This was done to best capture the resident who performed the work of patient care. In our institution, residents who merely "eyeball" a patient near the end of their shifts do not perform any documentation. A resident feeling comfortable dictating the chart generally has been involved in the decision making and work of caring for the patient. No credit was given to residents for patients inherited in signout. Off-service rotators, that is, non-EM residents working in the ED, were excluded from this study.

\section{Data analysis}

Productivity was calculated for all resident shifts by dividing the number of patients credited to the resident by the number of shift hours devoted to patient care. In a 12 -h shift, the denominator was therefore 12 , as all $12 \mathrm{~h}$ are devoted to picking up new patients, and residents complete their paperwork outside of their scheduled shift times. For 9-h shifts, the denominator was 8 , as residents devote $8 \mathrm{~h}$ to patient care and $1 \mathrm{~h}$ to completion of paperwork. If a resident has no paperwork to complete, he or she may leave after $8 \mathrm{~h}$. No resident during the study period initiated care on a patient beyond the eighth hour. Analysis of variance (ANOVA) was performed to assess for differences in productivity by shift number. Each resident's first shift in a series was designated " 1 ," the second consecutive shift was designated " 2 ," and so on.

\section{Results}

Productivity was tallied for 188 first-year shift strings, 303 second-year shift strings, and 224 third-year shift strings. First-year residents worked 12-h shifts exclusively. 
Second-year residents worked 101 12-h shifts and 163 9-h shifts. Third-year residents worked 156 12-h shifts and 37 9-h shifts. In the 3-month study period, firstyear residents worked 81 single shifts, 70 strings of 2 consecutive shifts, 27 strings of 3 consecutive shifts, 3 strings of 4 consecutive shifts, 3 strings of 5 consecutive shifts, 2 strings of 6 consecutive shifts, and 1 each of 7 consecutive shifts and 8 consecutive shifts. Firstyear residents saw $0.82,0.81,0.91,1.31$, and 1.03 patients per hour on their first five consecutive shifts $\left(F_{(2,175)}=2.89, p=0.06\right)$. We did not use the last two values for our ANOVA, as the $N$ for each was only 3 , and we did not wish to skew our results. Second-year residents worked 116 single shifts, 94 strings of 2 consecutive shifts, 60 strings of 3 consecutive shifts, 26 strings of 4 consecutive shifts, 6 strings of 5 consecutive shifts, and 2 strings of 6 consecutive shifts. Second-year residents saw $1.12,1.08,1.17,1.28,1.38$, and 1.29 patients per hour on their shifts, respectively $\left(F_{(3,292)}=4.19, p=0.006\right)$. We did not include the last two values in analysis, as the $N$ for these was 6 and 2. Third-year residents worked 111 single shifts, 65 strings of 2 shifts, 38 strings of 3 shifts, 7 strings of 4 shifts, and 1 string of 5 shifts. They saw 1.19, 1.24, $1.33,1.53$, and 1.42 patients per hour during those shifts $\left(F_{(2,211)}=4.08, p=0.02\right)$. We did not include the last two values in our data analysis, as the $N$ for a string of 4 shifts was 7 , and the $N$ for a string of 5 shifts was 1 .

\section{Discussion}

This study suggests that instead of tiring, residents maintain or improve productivity over consecutive shifts. Productivity improved reliably over the course of each consecutive shift for third-year EM residents. First- and second-year EM residents' overall productivity improved over the course of at least three consecutive shifts. The data collection involving second-year resident productivity afforded enough data points to analyze a fourth consecutive shift, which shows the same upward trend as the third consecutive shifts of the other two residency classes.

Why are residents more productive after consecutive shifts? There may be a practice effect that contributes to productivity. After working a shift, a resident may learn or relearn small things that improve their efficiency each day, and perhaps forget them when not working. Or, residents may become more confident in their abilities as they work through more patients and be able to manage more patients effectively. Perhaps there is an internal competitiveness, with residents wanting to see more patients each day to reach their personal best.

Dula et al. have shown that cognitive ability declines over the course of consecutive overnight shifts [12].
However, all forms of consecutive shifts, with strings of shifts including overnights, were part of this data set, and overall productivity improved. We did not perform any cognitive testing on our residents, but one would expect if cognitive decline was present, productivity would also decline. Additionally, the resident schedule was not based on a paradigm of set days or set shifts. Eventually, the shifts worked became fairly random in regards to day of the week, type of shift, or time of day but generally were modeled in a circadian fashion, that is, a resident would progress from a day shift to an evening shift to an overnight shift, and then have a day or two off before resuming a day or evening shift. We did not seek productivity differences in residents working a single shift type, for example, all evening shifts, in comparison to those working any combination of rapidly cycling shifts. Nor did we assess what rotation the resident had been on the prior month to see if there was a large practice effect after being off service for a month and returning to the ED or how many shifts back in the ED it would take to be as productive as peers who had been in the ED for consecutive months.

This study measured productivity as patients seen per hour. Some prior productivity literature has used relative value units (RVUs) as a measure of work productivity. The institution hosting this study utilizes electronic patient tracker software for the ED, but the system that calculates relative value units is not compatible with the tracker software. The system that calculates relative value units does so for attending physicians only and does not allow for tracking resident work productivity. By defining productivity as patients per hour, potential error was reduced secondary to fewer variables in calculating productivity. Jasani et al. recently showed that relative value units and productivity as measured by patients seen per hour correlate in a linear relationship [15]. This would suggest that either measure would result in the same trend.

Our patient tracking software does not utilize an acuity score, so we did not include patient acuity in our analysis. The study encompassed all three residency classes, and at the study institution, the second-year residents traditionally are assigned to the incoming high acuity traumas as well as to nontraumatic ED patients, while first- and third-year residents typically evaluate nontraumatic ED patients. There have been no studies to see how productivity is affected by primarily managing trauma patients as compared to nontraumatic patients, but it would seem that managing numerous trauma patients might alter productivity as defined by patients per hour, as these patients often require time-consuming procedures. That said, residents working "trauma shifts" see any patient waiting to be seen if there is no trauma patient available, and these shifts showed the same productivity trends as non-trauma shifts. 
Limitations

This study did not examine sign-out burden or number and acuity of patients and tasks inherited from one shift to the next. This could in theory affect productivity, especially in the initial hours of a shift. In addition, our system does not enable us to calculate RVUs per hour for residents. We do not provide data based on shift length. Subgroup analyses were performed based on shift length which demonstrated the same results: residents working multiple consecutive shifts of either 9- or 12-h shifts showed no decrease in productivity over time and a trend toward increased productivity. However, residents occasionally work mixed strings combining both 9- and 12-h shifts to accommodate conference time and cover vacation scheduling, so we included both kinds of shifts in our data analysis to better approximate real working conditions. We also did not attempt to control for total number of shifts worked in a given month or include non-ED clinical time (for instance, EMS time or off-service time) in our fatigue analysis. We did not attempt to control for residents who rotated through a variety of shift times versus those who worked predominantly a single type of shift (i.e., those cycling rapidly versus those working a cluster of day shifts). This could significantly affect fatigue, as it has been shown in nurses that rapid cycling in schedules increased sleepiness at work and likelihood of car crashes [16]. Finally, this investigation examined data at a single academic institution and may not apply to all academic EDs or to community EDs.

\section{Conclusions}

These data suggest that residents are not less productive and may actually be more productive at the end of a string of consecutive shifts. Therefore, total patient flow in the emergency room may benefit from scheduling residents in strings of at least three to four consecutive shifts. However, the number of shifts for maximized productivity was not determined. There were a few residents who did more than three or four consecutive shifts, but the sample size was too small and the confidence interval became too wide to interpret the data appropriately. Further studies could explore the ideal number of shifts before productivity declines. Resident scheduling might also benefit from overlapping strings, such that one resident is on his or her third or fourth consecutive shift when another begins his or her first shift in a string.

Conflicts of interest None.

\section{References}

1. DeBehnke D, O'Brien S, Leschke R (2000) Emergency medicine resident work productivity in an academic emergency department. Acad Emerg Med 7(1):90-92

2. Brennan DF, Silvestri S, Sun JY et al (2007) Progression of emergency medicine resident productivity. Acad Emerg Med 14 (9):790-794

3. Lammers RL, Roiger M, Rice L et al (2003) The effect of a new emergency medicine residency program on patient length of stay in a community hospital emergency department. Acad Emerg Med 10(7):725-730

4. Deveau JP, Lorenz JE, Hughes MJ (2003) Emergency medicine work productivity and procedural accomplishment. J Am Osteopath Assoc 103(6):291-296

5. Dowd MD, Tarantino C, Barnett TM et al (2005) Resident efficiency in a pediatric emergency department. Acad Emerg Med 12:1240-1244

6. France DJ, Levin S, Hemphill R et al (2005) Emergency physicians' behaviors and workload in the presence of an electronic whiteboard. Int J Med Inform 74:827-837

7. Cushman JT, Witting MD (2003) Emergency medicine resident scheduling and patient exposure. Acad Emerg Med 10(7):816818

8. Jeanmonod R, Brook C, Winther M, Pathak S, Boyd M (2009) Resident productivity as a function of emergency department volume, shift time of day, and cumulative time in the emergency department. Am J Emerg Med 27:313-319

9. Taffinder NJ, McManus IC, Gul Y, Russell RC, Darzi A (1998) Effect of sleep deprivation on surgeons' dexterity on laparoscopy simulator. Lancet 352(9135):1191

10. Weinger MB, Ancoli-Israel S (2002) Sleep deprivation and clinical performance. JAMA 287(8):955-957

11. Papp KK, Stoller EP, Sage P, Aikens JE, Owens J, Avidan A, Phillips B, Rosen R, Strohl KP (2004) The effects of sleep loss and fatigue on resident-physicians: a multi-institutional, mixedmethod study. Acad Med 79(5):394-406

12. Dula DJ, Dula NL, Hamrick C, Wood GC (2001) The effect of working serial night shifts on the cognitive functioning of emergency physicians. Ann Emerg Med 38(2):152-155

13. Folkard S, Tucker P (2003) Shift work, safety and productivity. Occup Med (Lond) 53(2):95-101

14. Horrocks N, Pounder R (2006) Who's for five nine-hour shifts a week? Clin Med 6(5):440-442

15. Barrett M, Levine B, Reed J et al (2008) An analysis of resident productivity: correlation between patients per hour and relative value units per hour. Acad Emerg Med 15(5 Suppl 1):S132

16. Gold DR, Rogacz S, Bock N et al (1992) Rotating shift work, sleep, and accidents related to sleepiness in hospital nurses. Am J Public Health 82(7):1011-1014

Rebecca Jeanmonod earned a BS in biology and philosophy at the University of Scranton and MD from Boston University. She did her residency in Emergency Medicine at Baystate Medical Center and served as Assistant Professor 2003-2008 at Albany Medical College and as Assistant Residency Director 2007-2008 at Albany Medical College. She is Assistant Professor at St. Luke's Hospital in Bethlehem, PA, and lives and works in Pennsylvania with her husband and four children. 